\title{
ATENÇÃO QUALIFICADA NOS CUIDADOS EM HIPERTENSÃO E DIABETES NA ESTRATÉGIA DE SAÚDE DA FAMÍLIA
}

\author{
Tamires Patrícia SOUZA ${ }^{1}$ \\ Rafaela SOUZA ${ }^{2}$ \\ Elisiane BISOGNIN ${ }^{3}$
}

\begin{abstract}
${ }^{1}$ Enfermeira. Especialista em Saúde Pública- Ênfase em Estratégia de Saúde da Família. Mestranda em Ciências Pneumológicas pela Universidade Federal do Rio Grande do Sul (UFRGS). tamires_psouza@ yahoo.com;

${ }^{2}$ Nutricionista. Residente em Sistema Público de Saúde - Ênfase em Atenção Básica pela Universidade Federal de Santa Maria (UFSM).rafa_nutri@yahoo.com.br;

${ }^{3}$ Enfermeira Obstetra. Especialista em Saúde da Família. elisbisognin@ gmail.com.
\end{abstract}

\section{Recebido em: 03/03/2015 - Aprovado em: 02/07/2015 - Disponibilizado em: 15/07/2015}

RESUMO: As Doenças Crônicas Não Transmissíveis (DCNT) representam um dos principais desafios de saúde, pois ameaçam a qualidade de vida de muitas pessoas, influenciando inclusive na economia dos países. Essa preocupação deve-se ao fato das DCNT serem passíveis de prevenção, e/ou tratamento. Buscou-se conhecer a realidade dos hipertensos e diabéticos em um município da região noroeste do estado do Rio Grande do Sul, referentes à Estratégia de Saúde da Família-2 (ESF-2), que atende cerca de 2053 pessoas. Teve como objetivo qualificar a atenção em saúde aos hipertensos e diabéticos. O estudo foi realizado nos meses de fevereiro a setembro do ano de 2012 e pode ser caracterizado como uma pesquisa ação. Os resultados proporcionaram maior entendimento da importância do cadastramento e acompanhamento dos hipertensos e diabéticos da área de abrangência da ESF-2. Para adequado seguimento do atendimento, é imprescindível que seja disponibilizado espaços para a capacitação da atuação dos profissionais não só aos hipertensos e diabéticos, mas sim a toda a população que necessita desse serviço. Conclui-se que as atividades da ESF devem ser baseadas em trabalho em equipe, contando com o apoio dos gestores de saúde, todos os profissionais envolvidos, bem como com o conhecimento e participação dos usuários do serviço.

Palavras-chave: Hipertensão. Diabetes. Estratégia de Saúde da Família. Sobrepeso. Doenças crônicas.

\section{WARNING QUALIFIED IN CARE IN HYPERTENSION AND DIABETES IN THE FAMILY HEALTH STRATEGY}

\begin{abstract}
Chronic Noncommunicable Diseases (NCDs) are main health challenges by threatening the quality of life of many people, including influencing the economy of countries. This concern is due to the fact that NCDs are preventable and / or treatment. We sought to know the reality of hypertension and diabetes in a municipality in the northwest region of the state of Rio Grande do Sul, referring to the Family Health Strategy-2 (FHE-2), which serves about 2053 people. The objective was to qualify the health care for hypertensive and diabetic. The study was conducted from February to September of the year 2012 and can be characterized as an action research. The results provided greater understanding of the importance of registration and monitoring of hypertension and diabetes in the coverage area of the FHE-2. For appropriate follow-up care, it is essential that is made available spaces for training the work of professionals not only to hypertension and diabetes, but the entire population that needs this service. We conclude that the activities of the ESF should be based on teamwork, with the support of health care managers, all professionals involved, as well as with the knowledge and participation of service users.
\end{abstract}

Keywords: Hypertension. Diabetes. Family Health Strategy. Overweight. Chronic Diseases. 


\section{INTRODUÇÃO}

As Doenças Crônicas Não Transmissíveis (DCNT), em especial a hipertensão arterial e o diabetes representam um dos principais desafios de saúde para o desenvolvimento global nas próximas décadas (BRASIL, 2012). Por meio da vigilância em saúde é possível monitorar e analisar o perfil das doenças e de seus fatores determinantes e condicionantes, bem como detectar mudanças nas suas tendências no tempo, no espaço geográfico e em grupos populacionais. O MS refere que um indicador a ser considerado é que essas doenças aumentam mais depressa em países pobres e em desenvolvimento, sendo um impacto negativo em razão da morbimortalidade precoce (BRASIL, 2006).

No Brasil, as duas patologias Diabetes Mellitus (DM) e Hipertensão Arterial Sistêmica (HAS), são responsáveis pela primeira causa de mortalidade e de hospitalizações, de amputações de membros inferiores e representa ainda $62,1 \%$ dos diagnósticos primários em pacientes com Insuficiência Renal Crônica (IRC) submetidos à hemodiálise (BRASIL, 2006). Segundo Rocha (2001), a principal causa de insucesso no tratamento é sua não adesão. O que de certa forma, foi pensado ao estabelecer e implementar o programa HIPERDIA/MS, que, segundo Machado (2002), é a maior proposta de intervenção sobre essas duas doenças crônicas e demais fatores de risco para doenças cardiovasculares já realizadas no Brasil.

Dessa maneira, os autores Lima et al., (2004) e Ximenes et al., (2005), defendem que para um controle adequado da HAS e da DM, são necessários mais do que medidas de orientação, mas também estratégias que ajudem os indivíduos na mudança das atitudes diárias de vida, alimentação; Ações de promoção à saúde e prevenção das doenças. Portanto, para o acompanhamento, torna-se fundamental um controle sistemático desses indivíduos, especialmente na atenção básica à saúde, como o auxílio das fontes de informação sobre os portadores desses agravos.

Assim, verificando a ineficácia dos sistemas de atendimento ao hipertenso e diabético, devido à forma como estavam sendo implementados os programas de atenção, com baixa adesão aos grupos e consultas periódicas, bem como pela baixa adesão ao cadastro e alimentação do programa HIPERDIA optou-se por desenvolver este estudo em um município da região noroeste do estado do Rio Grande do Sul.

Por se tratar de um projeto-ação, objetivou-se qualificar a atenção em saúde aos hipertensos e diabéticos da Estratégia de Saúde da Família - 2 do município estudado, identificando os casos conhecidos e novos casos de HAS e DM; mapear os pacientes de alto risco; realizar acompanhamento periódico, com consultas, exames e visitas 
domiciliares; realizar avaliação clínica de acordo com o preconizado pelo Ministério da Saúde e criar roteiro prático de consulta.

\section{METODOLOGIA}

O estudo foi realizado nos meses de fevereiro a setembro do ano de 2012, e pode ser caracterizado como uma pesquisa-ação, descrita por Minayo (1996), como um tipo de investigação social com base empírica que é concebida e realizada com o intuito de organizar uma ação ou uma resolução de um problema coletivo, onde pesquisadores e participantes estão envolvidos de modo cooperativo ou participativo.

Os sujeitos da pesquisa foram pessoas portadoras de hipertensão e/ou diabetes que moravam na área pertencente a ESF-2 do município em questão.

O município, de aproximadamente 7.500 habitantes (IBGE, 2014), conta com 3 ESFs, tendo cobertura de $100 \%$ da população, tanto da zona rural, quanto da zona urbana. A ESF-2 tem abrangência na região norte e noroeste do município, atingindo parte da zona urbana e parte da zona rural e atende cerca de 2053 pessoas. Sendo que, a ESF-2 possui 296 hipertensos, destes, 41 também são diabéticos.

Foi encaminhado à instituição uma Carta de Apresentação do projeto de intervenção a fim de adquirir a autorização da
Instituição. O projeto faz parte de um projeto guarda-chuva, e por esse motivo não possui numero de liberação do Comitê de Ética em Pesquisa da Universidade Federal de Pelotas UFPEL. O início da pesquisa deu-se após a autorização do CEP.

Para a coleta de dados, foram realizadas análises documentais e observação participante através de visitas ao local da pesquisa. Os dados foram inseridos em planilha eletrônica disponibilizada pela UFPEL e codificados, sendo possível a partir de então a análise dos dados, seguindo os seguintes passos para organização dos dados: agrupamento e resumo dos dados através de tabelas de frequências, resumo das principais estatísticas, análise e interpretação dos dados, análise de correlação entre os dados.

A intervenção foi baseada no programa HIPERDIA do MS, e para dar início à intervenção foram realizadas reuniões com a equipe que já estava atuando na ESF-2. Eram avaliados 30 usuários por dia, divididos entre 15 pela manhã e 15 pela tarde, todas as segundas e quintas-feiras, exceto nos feriados.

No $4^{\circ}$ mês de intervenção foram iniciadas os grupos de educação continuada para os pacientes e profissionais da equipe da ESF-2. 


\section{RESULTADOS E DISCUSSÃO}

No desenvolvimento das ações do projeto de intervenção procurou-se envolver todas as partes interessadas desde os gestores, profissionais da ESF, mas principalmente a população estudada, já que de acordo com Filha et al., (2011), para avaliar um programa de atenção à saúde da magnitude do HIPERDIA é imprescindível envolver o usuário, como sujeito partícipe do processo e cidadão portador de direitos.

Ao contrário do estudo realizado por Filha et al., (2011), onde os resultados revelaram que os hipertensos e diabéticos na sua maioria realizavam consultas médica e de enfermagem de forma semestral, com exames laboratoriais anuais. Na ESF estudada, a realidade era modificada no contexto de acompanhamento periódico desses pacientes, sendo que os hipertensos e diabéticos, procuravam o serviço de saúde em questão, por motivos diversos, e não ao seu agravo principal.

Comparando-se ainda o estudo realizado por Filha et al., (2011), pode-se avaliar que haviam lacunas na realização de consultas, exames, bem como na oferta de medicação antes da realização do projeto de intervenção. Sugerindo como alternativa da autora supracitada, que os profissionais da ESF focalizem atentamente estes pacientes, estabelecendo dias prioritários para seu atendimento, facilitando o acesso desses pacientes aos serviços disponíveis.

Nesse sentido, com o desenvolvimento da intervenção do projeto, pode-se observar um avanço no atendimento aos HAS e DM, pois facilitou a organização do atendimento na ESF. Foi possível estender a cobertura de atendimento a $99 \%$ dos HAS e DM, sendo todos acompanhados pelos quatro meses de intervenção, e programação para acompanhamento periódico a esses pacientes. Foi possível atualizar os exames preventivos desses pacientes, bem como a adequação das medicações utilizadas.

A partir desta observação, pode-se agora discorrer sobre os achados da pesquisa. Foram estudados 296 pacientes diagnosticados anteriormente com HAS, e 41 destes pacientes, também diagnosticados com DM. Identificou-se predomínio de pacientes do sexo feminino em ambos os grupos, totalizando 207 (70\%) dos pacientes hipertensos, e 27 (66\%) dos pacientes diabéticos. Com relação à idade dos hipertensos, houve predomínio da faixa etária de 50 a 59 anos, com 29 pacientes (10\%) do sexo masculino, e no sexo feminino, o predomínio deu-se no mesmo intervalo de 50 a 59 anos, com 65 pacientes (22\%).

Quando analisou-se a faixa etária dos pacientes diabéticos, observou-se distribuição idêntica nos intervalos de 50 a 59 anos e 60 a 69 anos, com 5 pacientes do sexo masculino em cada. No sexo feminino, sobressaiu-se a 
faixa etária de 60 a 69 anos, com 12 pacientes estudadas (29\%) (Tabela 1). As informações obtidas concordam com pesquisas diversas onde predominam os casos de HAS e DM em pessoas adultas e idosas, de ambos os sexos.

O diagnóstico de hipertensão arterial se torna mais comum com a idade avançada, alcançando cerca de $8 \%$ dos indivíduos entre os 18 e os 24 anos de idade e mais de $50 \%$ na faixa etária de 55 anos ou mais de idade. Dados do MS também apontam que o diagnóstico de hipertensão é maior em mulheres (25\%) do que em homens (21\%) (BRASIL, 2011).

Tabela 01- Características da população.

\begin{tabular}{|c|c|c|c|c|}
\hline \multirow{2}{*}{ Variáveis } & \multicolumn{2}{|c|}{ Hipertensos } & \multicolumn{2}{|c|}{ Diabéticos } \\
\hline & $\mathbf{n}$ & $\%$ & $\mathbf{n}$ & $\%$ \\
\hline \multicolumn{5}{|l|}{ Sexo } \\
\hline Masculino & 89 & $30 \%$ & 14 & $34 \%$ \\
\hline \multicolumn{5}{|l|}{ Idade } \\
\hline$<39$ & 0 & 0 & 0 & 0 \\
\hline $40-49$ & 7 & $2 \%$ & 1 & $2 \%$ \\
\hline $50-59$ & 29 & $10 \%$ & 5 & $12 \%$ \\
\hline $60-69$ & 21 & $7 \%$ & 5 & $12 \%$ \\
\hline $70-79$ & 18 & $6 \%$ & 4 & $10 \%$ \\
\hline$>80$ & 7 & $2 \%$ & 1 & $2 \%$ \\
\hline \multicolumn{5}{|l|}{ Sexo } \\
\hline Feminino & 207 & $70 \%$ & 27 & $66 \%$ \\
\hline \multicolumn{5}{|l|}{ Idade } \\
\hline$<39$ & 3 & $1 \%$ & 0 & 0 \\
\hline $40-49$ & 29 & $10 \%$ & 1 & $2 \%$ \\
\hline $50-59$ & 65 & $22 \%$ & 6 & $15 \%$ \\
\hline $60-69$ & 55 & $18 \%$ & 12 & $29 \%$ \\
\hline $70-79$ & 32 & $11 \%$ & 6 & $15 \%$ \\
\hline$>80$ & 15 & $5 \%$ & 2 & $5 \%$ \\
\hline
\end{tabular}

Ainda comparando a pesquisa com dados encontrados na literatura, a prevalência de mulheres cadastradas como hipertensas e diabéticas também são visualizados em outros municípios, como no estudo realizado nos sistemas de informações em saúde e de cadastramento do país o HIPERDIA, apontando que em todas as faixas etárias há mais mulheres, sendo a maior diferença na idade entre 40 e 49 anos. No ano de 2007, foram cadastradas 387.754 pacientes no Sistema, sendo 66\% (255.830) de mulheres e $34 \%$ (131.924) de homens (BOING \& BOING, 2007).

Em dados apontados pelo VIGITEL Brasil (2011), a proporção de adultos diagnosticados como portadores de DM variou de 5\% para homens e $6 \%$ para mulheres, levando em consideração que são as mulheres que mais procuram os serviços de saúde, o que talvez seja responsável pelas estatísticas apontar mais mulheres acometidas pelos dois agravos estudados. Somado a essas duas comorbidades, ainda existem alguns agravos identificados na população, dentre eles, sedentarismo, tabagismo e sobrepeso (Tabela 02). 
Tabela 02: Agravos associados à hipertensão e diabetes na população atendida pela ESF 02.

\begin{tabular}{lcrcc}
\hline \multirow{2}{*}{ Variáveis } & \multicolumn{2}{c}{ Hipertensos } & \multicolumn{2}{c}{ Diabéticos } \\
\cline { 2 - 5 } & \multicolumn{1}{c}{$\mathbf{N}$} & $\%$ & $\mathbf{N}$ & $\%$ \\
\hline Tabagismo & & & & \\
Sim & 126 & $42 \%$ & 6 & $15 \%$ \\
Não & 170 & $57 \%$ & 35 & $85 \%$ \\
\hline Sedentarismo & & & & \\
Sim & 171 & $58 \%$ & 29 & $71 \%$ \\
Não & 125 & $42 \%$ & 12 & $29 \%$ \\
\hline Sobrepeso & & & & \\
Sim & 224 & $76 \%$ & 34 & $83 \%$ \\
Não & 72 & $24 \%$ & 7 & $17 \%$ \\
\hline
\end{tabular}

Fonte: As autoras.

Acompanhando também, os dados estatísticos apresentados pelo VIGITEL no ano 2011, o excesso de peso foi diagnosticado em cerca de $52 \%$ dos homens e $45 \%$ das mulheres portadoras de DM. De acordo com a pesquisa realizada, $76 \%$ dos pacientes hipertensos apresentam também sobrepeso associado, e $83 \%$ dos pacientes diabéticos também estão com sobrepeso.

Estudos de Corrêa et al. (2006), afirmam que a redução de peso e a manutenção do peso adequado com IMC entre 20 a $25 \mathrm{~kg} / \mathrm{m}^{2}$ diminuem de forma significativa a PA e previnem o aparecimento da hipertensão arterial. Com relação aos idosos ocorre o contrario, o gênero masculino se mantém com IMC adequado enquanto que o feminino passa para o sobrepeso.

O sedentarismo se tornou nos dias atuais um grande problema de saúde pública, e sabidamente aumenta a incidência de hipertensão arterial em $30 \%$ quando comparados aos ativos (FAGARD, 2005). Em amostras da nossa população, a combinação de fatores de risco entre indivíduos hipertensos parece variar com a idade, predominando a inatividade física e o sobrepeso.

Embora a prevalência do tabagismo tenha sido encontrada em menor quantidade nos pacientes, tanto hipertensos, quanto diabéticos, sabe-se que evitar esse hábito, é um dos maiores desafios em razão da dependência química causada pela nicotina. No entanto, de acordo com Viegas et al., (2004), o tabagismo é o único fator de risco totalmente evitável de doença e morte cardiovasculares.

\section{CONCLUSÃO}

Com o presente trabalho pode-se portanto, identificar os casos conhecidos e novos casos de pacientes com HAS e DM da ESF 2. Mapear os pacientes de alto risco e realizar acompanhamento periódico, com consultas, exames e visitas domiciliares. Realizar avaliação clínica de acordo com o preconizado pelo Ministério da Saúde.

Assim, foi possível a compreensão da importância do cadastramento e acompanhamento dos hipertensos e diabéticos da área de abrangência da ESF 2 no que se refere a visualização dos casos mais graves, controle mais rigoroso do acompanhamento, 
bem como na mensuração dos pacientes acometidos pelos dois agravos estudados.

Conclui-se, portanto, que as atividades da ESF devem ser baseadas em trabalho em equipe, contando com o apoio dos gestores de saúde, todos os profissionais envolvidos, bem como com o conhecimento e participação dos usuários, pois pode-se perceber, que a partir do momento que envolvem-se todos esses pontos em um mesmo trabalho, os resultados são positivos.

\section{REFERÊNCIAS}

BRASIL, Ministério da Saúde. Vigilância de Doenças Crônicas Não Transmissíveis (DCNT). 2012.

Ministério da Saúde. Hipertensão arterial atinge $23,3 \%$ dos brasileiros. Disponível em $<$ http://portal.saude.gov.br/portal/aplicacoes/n oticias/default.cfm?pg=dspDetalheNoticia\&id _area $=124 \&$ CO_NOTICIA $=12485>$. Acesso em: 26/04/2011.

Ministério da Saúde. VIGITEL BRASIL 2011: Saúde Suplementar. Vigilância de Fatores de Risco e Proteção para Doenças Crônicas por Inquérito Telefônico. Rio de Janeiro, RJ 2012.

Ministério da Saúde. Cadernos de Atenção Básica no 15: Hipertensão Arterial Sistêmica. Brasília, 2006.

BOING, A.C.; BOING, A.F. Hipertensão arterial sistêmica: o que nos dizem os sistemas brasileiros de cadastramentos e informações em saúde. Revista Brasileira de Hipertensão vol.14(2): 84-88, 2007.

CORRÊA, Thiago, SILVA, Camila, CASTRO, Melina. et aL. Hipertensão arterial sistêmica:atualidades sobre sua epidemiologia, diagnostico e tratamento.Artigo de revisão.Med.ABC. São Paulo. 2006.

FAGARD, R.H. Physical activity, physical fitness and the incidence of hypertension. $\mathrm{J}$ Hypertens

2005;23:265-7.

FILHA， F.S.S.C.; NOGUEIRA， L.T.; VIANA, L.M.M.; HIPERDIA: adesão e percepção de usuários acompanhados pela Estratégia de Saúde da Família. Revista Rene, Fortaleza, 2011.

IBGE, Diretoria de Pesquisas, Coordenação de População e Indicadores Sociais.

NOTA 1: Estimativas da população residente com data de referência 10 de julho de 2014 publicadas no Diário Oficial da União em 28/08/2014.

LIMA, M.T.; BUCHER, J.S.N.F.; LIMA, J.W.O. A hipertensão arterial sob o olhar de uma população carente: estudo exploratório a partir dos conhecimentos, atitudes e práticas. Cadernos de Saúde Publica. 2004. Disponível em:

$<$ http://www.scielosp.org/scielo.php?script=sc i_pdf\&pid=S0102-

311X2004000400023\&lng=es\&nrm=iso\&tln $\mathrm{g}=\mathrm{pt}>$. Acesso em: 15/11/2011.

MACHADO, C.A. Palavra do presidente. Revista Brasileira de Hipertensão.2002.

MINAYO, M. C. S. O desafio do conhecimento. Rio de Janeiro: Hucitec. 1996.

ROCHA, J.C. Prefácio. In: NOBRE, F.; PIERUN, A.M.G.; MION, J.D. Adesão ao tratamento: o grande desafio da hipertensão. São Paulo: Lemos Editorial. 2001.

VIEGAS, C.A.A.; ARAÚJO, A.J.; MENEZES, A.M.B.; DÓREA, A.J.P.; TORRES, B.S. Diretrizes para cessação do tabagismo. J Bras Pneumol 2004;30(Sup12):S1-S76. 
XIMENES, N.F.R.; MELO, J.R. Controle da hipertensão arterial na atenção primária em saúde - uma análise das práticas do enfermeiro. Enfermeria Global. 2005.
Disponível

em: $<$ http://revistas.um.es/index.php/eglobal/articl e/viewFile/506/552>. Acesso em: 15/11/2011. 